\title{
DETERMINATION OF THE OPTIMAL OPERATING PARAMETERS FOR THE JEFFERSON LAB'S CRYOGENIC COLD COMPRESSOR SYSTEM
}

\author{
J. D. Wilson, Jr., V. Ganni, D. M. Arenius, J. D. Creel \\ Thomas Jefferson National Accelerator Facility, Cryogenics Group \\ Newport News, Virginia, 23606, USA
}

\begin{abstract}
Jefferson Lab's (JLab) Continuous Electron Beam Accelerator Facility (CEBAF) and Free Electron Laser (FEL) are supported by $2 \mathrm{~K}$ helium refrigerator known as the Central Helium Liquefier (CHL), which maintains a constant low vapor pressure over the accelerators' large liquid helium inventory with a five-stage centrifugal compressor train. The cold compressor train operates with constrained discharge pressure and can be varied over a range of suction pressures and mass flows to meet the operational requirements of the two accelerators. Using data from commissioning and routine operations of the cold compressor system [1], the presented procedure predicts an operating point for each cold compressor such that maximum efficiency is attained for the overall cold compressor system for a given combination of mass flow and vapor pressure. The procedure predicts expected efficiency of the system and relative compressors speeds for operating vapor pressures from 4 to $2.5 \mathrm{kPa}$ (corresponds to overall pressure ratios of 29 to 56) and flow rates of $135 \mathrm{~g} / \mathrm{s}$ to $250 \mathrm{~g} / \mathrm{s}$. The results of the predictions are verified by test for a few operating conditions of mass flows and vapor pressures.
\end{abstract}

\section{INTRODUCTION}

Since 1994, CHL [2] with its cold compressors maintains the large 2 K liquid helium (LHe) inventory in each linear accelerator (LINAC). In June 1999, commissioning data was collected for the new series of five cold compressors, which are housed in the Redundant 2 K Cold Box (SCN) (FIGURE 1). The accelerators contain cryomodules with a total liquid volume of approximately 70,000 liters. All cryomodule vapor spaces are connected and controlled as a single volume. The magnitude of CHL's load is mostly determined by the electron beam energy needed to support the accelerators' schedule.

The saturated vapor from the accelerators flows to the inlet of the cold compressor system via a heat exchanger, HX-10N (FIGURE 2). Since SCN commissioning, the accelerators' operating pressure has been $4 \mathrm{kPa}$ with the selectable mass flow control set to 
maintain a constant value between 190 and $210 \mathrm{~g} / \mathrm{s}$. To support the accelerators' load requirements (vapor pressure and mass flow), CHL's control system follows some basic rules to ensure stable operations.

- Based upon experience with the original four-stage cold compressor system, the speed of CC4N is set for the desired mass flow. Speed automatically responds to flow variations.The electric heaters in the cryomodules automatically adjust to keep vapor pressure constant by compensating for changes in the dissipated radio frequency power.

- For all steady state operations, the relative speed ratios of $\mathrm{CC} 1 \mathrm{~N}, \mathrm{CC} 2 \mathrm{~N}$, and CC3N were constant with respect to CC4N speed.

- $\mathrm{CC} 3 \mathrm{~N}$ speed fraction relative to $\mathrm{CC} 4 \mathrm{~N}$ is 0.69

- $\mathrm{CC} 2 \mathrm{~N}$ speed fraction relative to $\mathrm{CC} 4 \mathrm{~N}$ is 0.365

- $\mathrm{CC} 1 \mathrm{~N}$ speed fraction relative to $\mathrm{CC} 4 \mathrm{~N}$ is 0.17

- The normal method of CC5N control is the automatic adjustment of speed to keep a constant pressure difference between CC5N inlet and outlet; typically $30 \mathrm{kPa}$.

- The alternative (rarely used) method of CC5N speed control is to maintain a constant relative speed with respect to $\mathrm{CC} 4 \mathrm{~N}$ speed.

\section{PROBLEM STATEMENT}

With no cost and negligible impact on the accelerator's schedule, the challenge was to develop a prediction method for the parameters associated with the maximum isentropic

\section{Main Compressors}

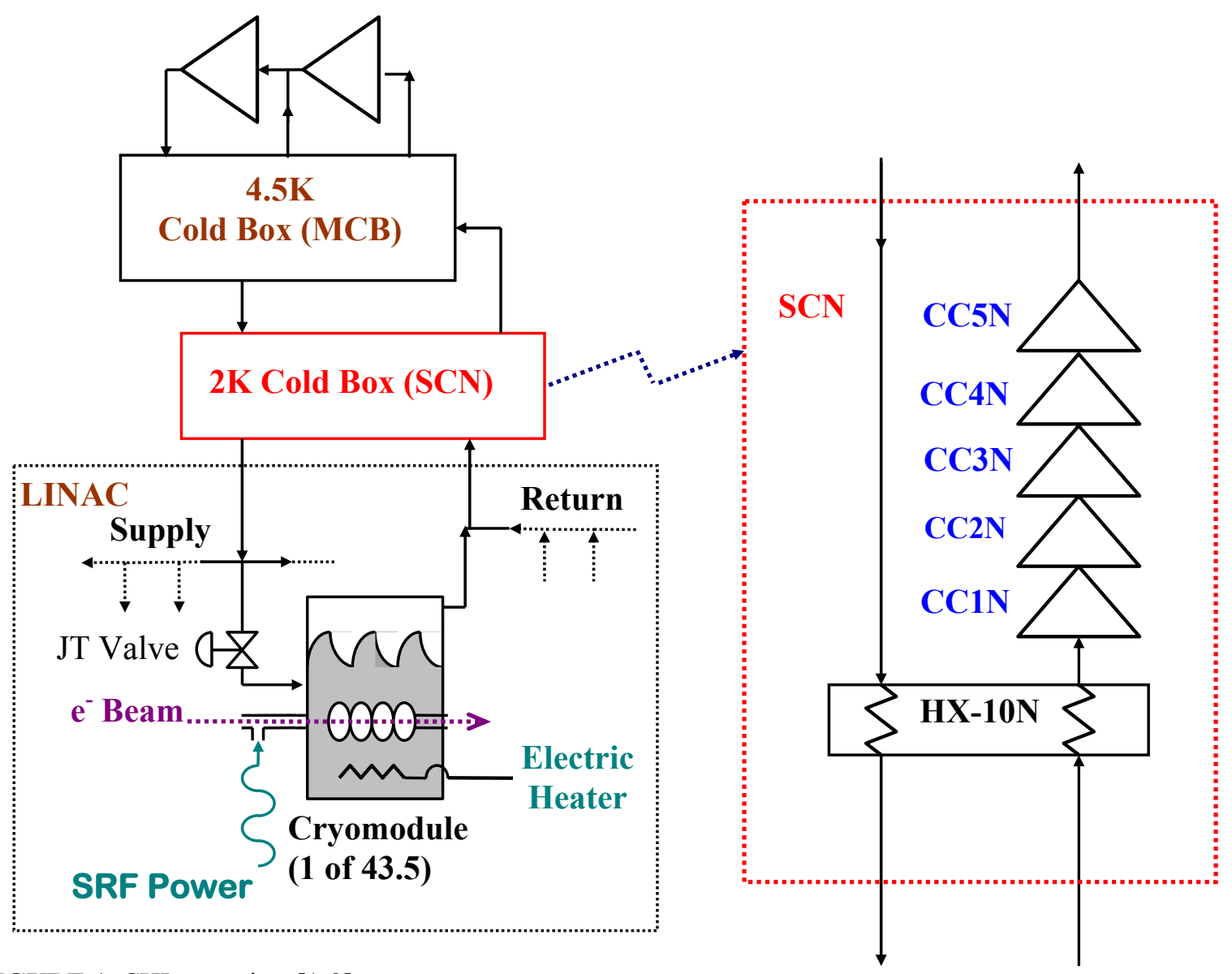

FIGURE 1. CHL overview [1-2]. 
efficiency of the overall cold compressor train. The technique for optimization was required to apply to a wide range of load conditions (combinations of vapor pressure and mass flow) as anticipated by future accelerator requirements.

\section{MODEL OVERVIEW}

The analysis was based on the commissioning data [1] for individual cold compressors. At constant load condition, the only adjustable parameters were the speeds of each radial centrifugal compressor. Along with other outputs, the optimization program calculated the rotational speed of each compressor required to support the predicted maximum attainable isentropic efficiency of the overall cold compressor train based upon a pair of inputs: mass flow and liquid helium saturation pressure [3]. The range of load conditions were flow rates of $135 \mathrm{~g} / \mathrm{s}$ to $250 \mathrm{~g} / \mathrm{s}$ and saturation pressures of 4 to $2.5 \mathrm{kPa}$.

The cold compressors' operational region was defined by recorded extremes in temperature and pressure at the cold compressor system's inlet and outlet. Within the region, the compressibility factor is very near unity (within five parts per million). Therefore, the ideal gas relationships were utilized.

\section{MODELING EQUATION DEVELOPMENT}

The model required characteristic equations (functions) to describe the cold compressor train's boundary conditions and the unique behavior of each radial compressor (APPENDIX A). The independent variables were the load condition and the specific speed for each compressor. For a given load condition (vapor pressure and mass flow), there exists an optimal set of specific speeds.

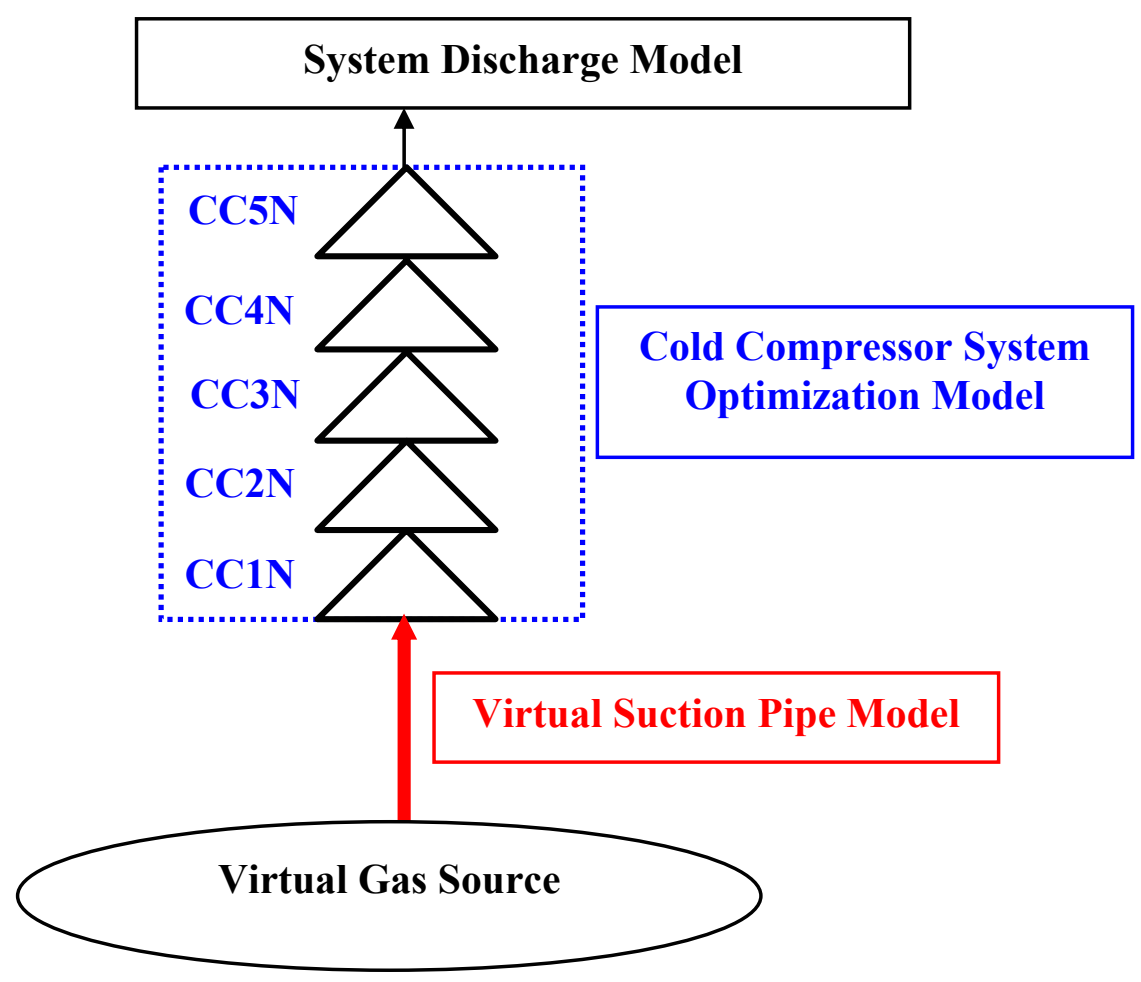

FIGURE 2. Cold compressor model boundaries [3]. 
For each compressor, the polynomial curve fitting of commissioning data provided two characteristic equations. These two functions of specific speed (independent variable) were polytropic efficiency (dependent variable) and head coefficient (dependent variable).

Since June 1999, JLab's operating schedule allotted only a few hours for saturation pressure below $4 \mathrm{kPa}$. The model accounted for vapor pressure changes by determining the boundary condition (FIGURE 1) of overall pressure ratio.

The system discharge pressure is fairly insensitive to discharge temperature. Therefore, the discharge pressure is a function of mass flow only. The suction pressure is a function of mass flow and vapor pressure. The combined behavior of the system inlet piping network and heat exchanger was simplified into a function called the "virtual suction pipe." The function permitted calculation of thermodynamic properties at the system inlet as a function of the load condition.

The detailed description of the model development was published in a separate document [3].

\section{MODEL RESULTS}

In plots of model results (FIGURES 3-5), the discontinuous slopes in both overall efficiency graphs and the individual compressor angular speeds graphs are caused by individual compressor limitations. The maximum allowed speed for $\mathrm{CC} 1 \mathrm{~N}$ limits the maximum attainable pressure ratio for $\mathrm{CC} 1 \mathrm{~N}$ at the low vapor pressures and high mass flows. Therefore, $\mathrm{CC} 2 \mathrm{~N}$ pressure ratio increases to compensate for $\mathrm{CC} 1 \mathrm{~N}$ 's decreasing contributing to the overall pressure ratio. Also, the model ensures stable operation of CC5N with a minimum allowed pressure ratio.

\section{TESTING}

During a couple of rare testing opportunities, the overall isentropic efficiency (FIGURE 6) improved when the relative speed fraction were increased according to the predicted values. The efficiency did not improve until CC1N relative speed ratio was increased after the other compressor speed adjustments. Through years of experience of maintaining the vapor pressure near $4 \mathrm{kPa}$, operators found a set of parameters for that result in an overall efficiency values which are near the model predictions for mass flow values between 190 and 215 grams per second.
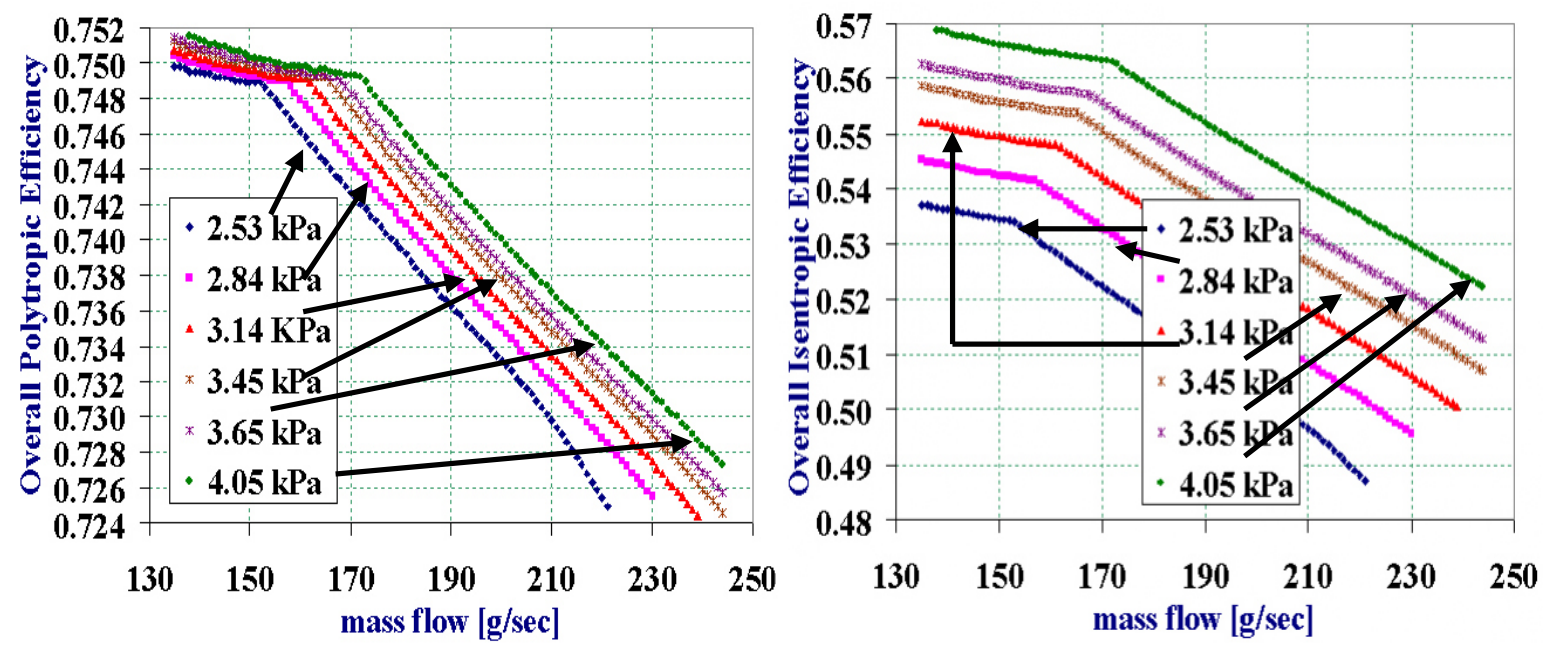

FIGURE 3. Predicted overall isentropic and polytropic efficiencies: function of load. 
During testing, high-indicated motor current imposed a limit on CC1N speed. The manufacturer's method for modulating electric power to the variable speed motors was unavailable. Therefore, the motor performance was excluded from the optimization model.
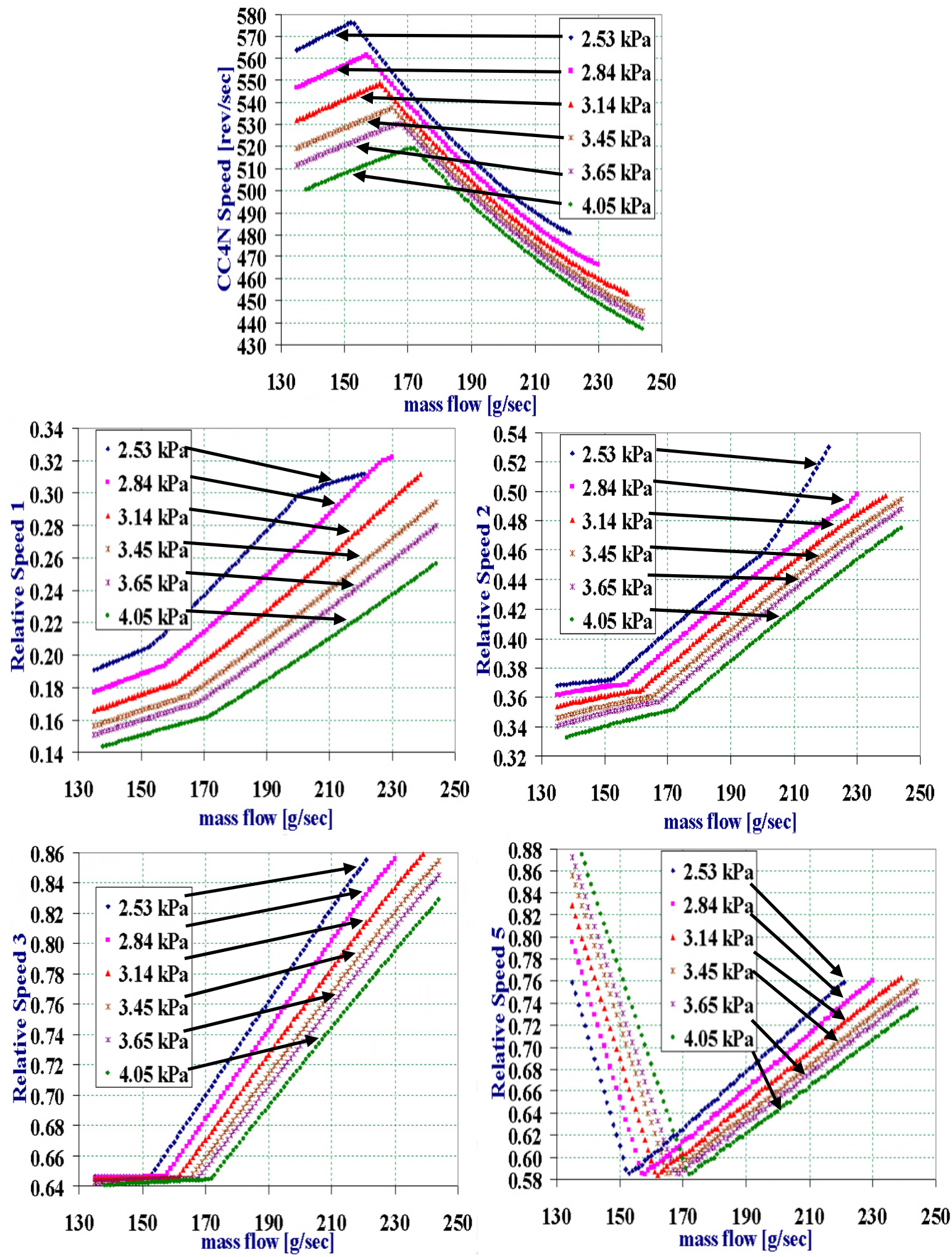

FIGURE 4. Predicted speed of CC4N and relative speeds of CC1N, CC2N, CC3N and CC5N: function of load. 


\section{CONCLUSION}

For the given wide range of load conditions, the optimization program solved the mathematical function that generates a value for maximum overall isentropic efficiency of the cold compressor system for each combination of mass flow value and saturation liquid helium pressure. The program output includes each compressor's rotational speed required
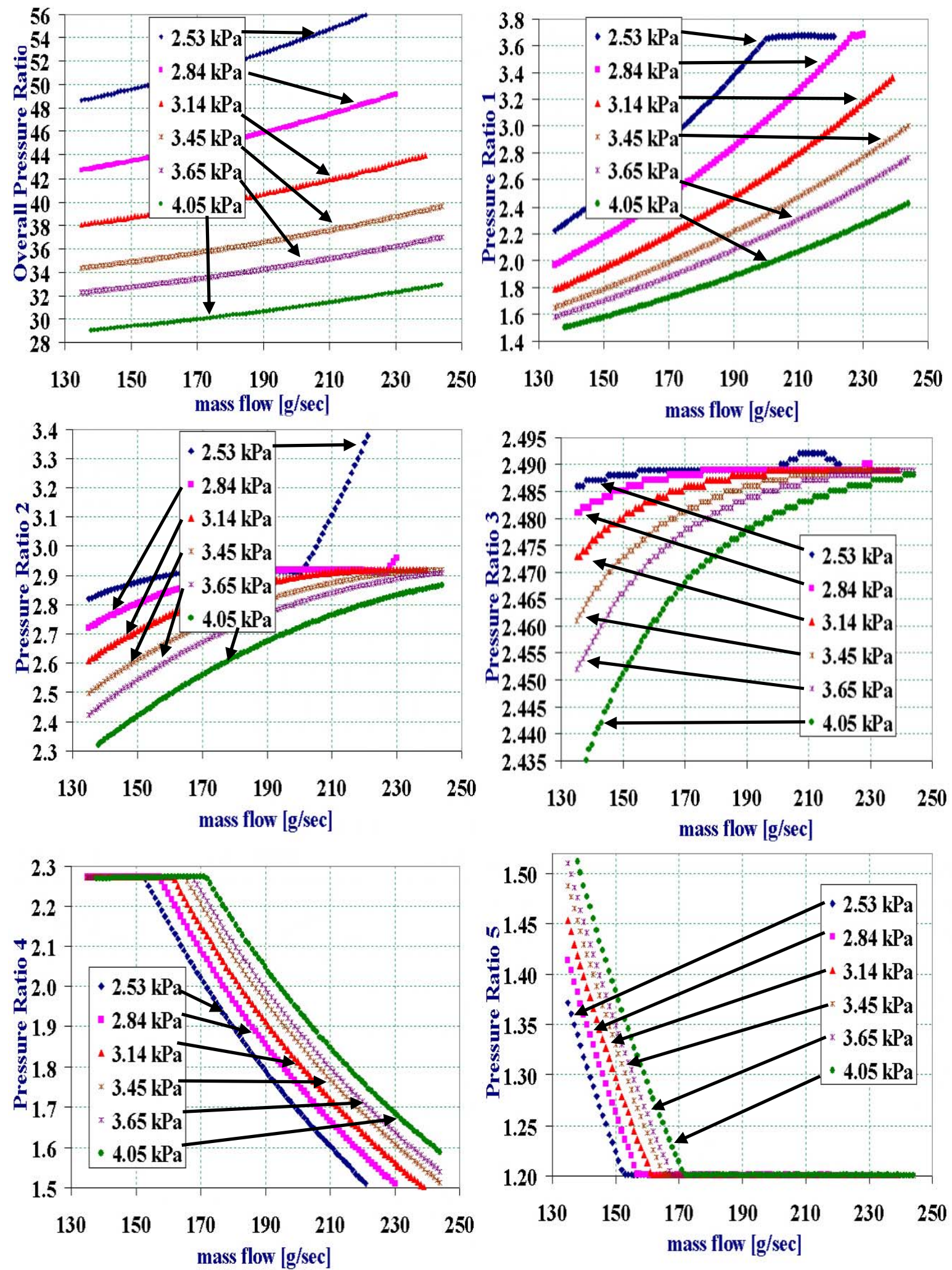

FIGURE 5. Predicted overall pressure ratio and individual compressor pressure ratios: function of load 

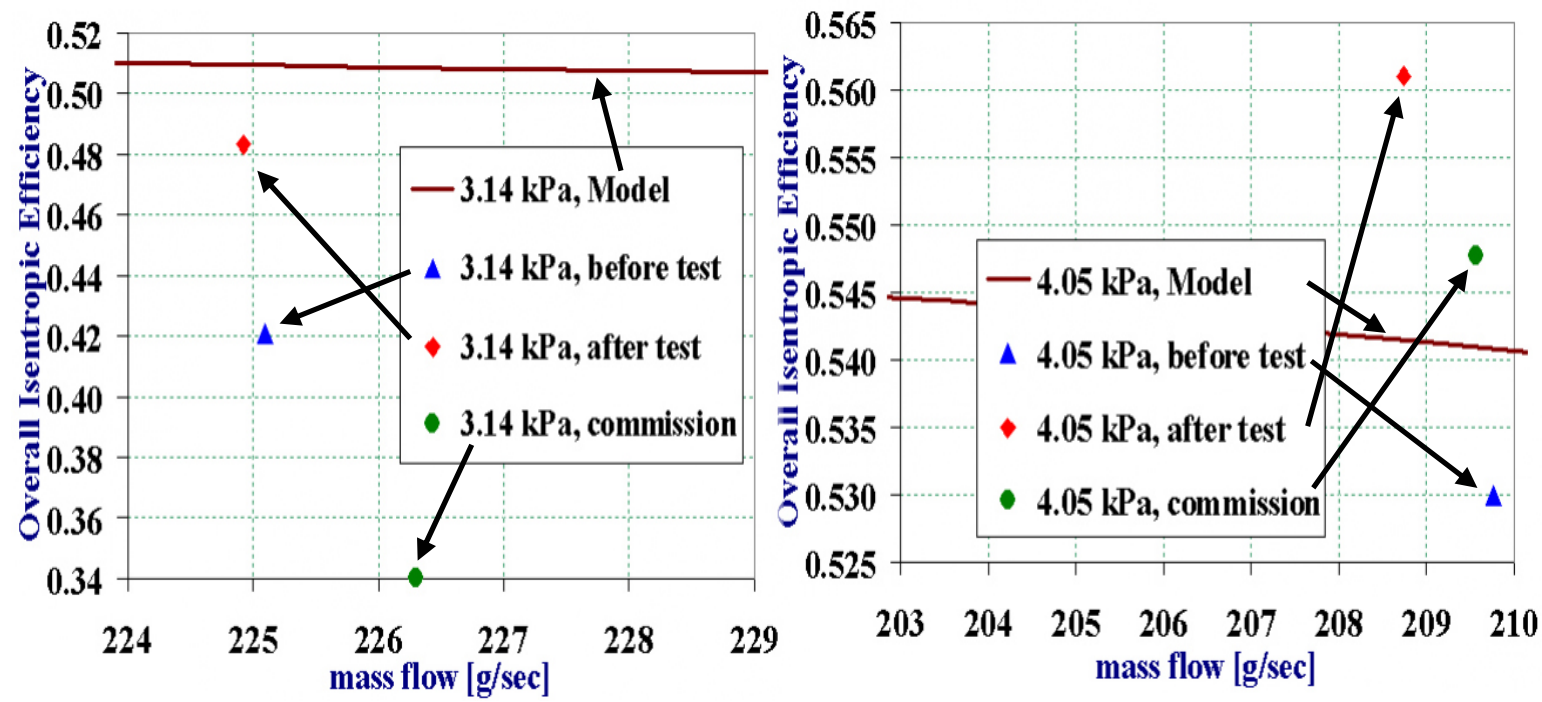

FIGURE 6. Test results for vapor pressures of $3.14 \mathrm{kPa}$ (16 Oct 02) and $4 \mathrm{kPa}(20 \mathrm{Feb} 02)$

to achieve the maximum efficiency state for each load condition. For future operations at lower vapor pressures and wide range of mass flows, the model results are especially valuable. Variable speed electric motor performance issues limited the attainability of the model predictions. The few testing opportunities revealed that significant efficiency improvements are attainable with a stable system by adjusting the relative speed of the first cold compressor to a value less than that predicted for maximum efficiency. The optimization model of the cold compressor system is a powerful tool for improving the capability and operating range of Jefferson Lab's Central Helium Liquefier.

\section{REFERENCES}

1. Ganni, V., Arenius, D. M., Bevins, B. S., Chronis, W. C., Creel, J. D., Wilson, J.D., Jr., "Design, Fabrication, Commissioning, and Testing of a 250 G/S, 2-K Helium Cold Compressor System," in Advances in Cryogenic Engineering 47A, edited S Breon et al., AIP Press, Melville, New York, 2002, pp. 288-304.

2. Chronis, W. C. et al, "Procurement and Commissioning of the CHL Refrigerator at CEBAF," in Advances in Cryogenic Engineering 41A, edited by P. Kittel, Plenum, New York, 1995, pp. 641-648.

3. Wilson, J.D. Jr., "Determination of the Optimal Operating Parameters for Jefferson Laboratory's Cryogenic Cold Compressor System," Master of Science Thesis, Christopher Newport University, Newport News, Virginia, 2003.

\section{APPENDIX-A}

Head

Pressure Ratio

Temperature Ratio

Flow Coefficient

$$
H=\frac{\Delta P}{\rho g}
$$$$
P_{r}=\frac{P_{\text {outlet }}}{P_{\text {inlet }}}
$$ 
Head Coefficient

Specific Speed

Polytropic Efficiency

Isentropic Efficiency

Mass Flow

\section{Where}

$D=$ Impeller Diameter (m)

$P=$ Pressure $(\mathrm{kPa})$

$T=$ Temperature $(\mathrm{K})$

$\omega=$ Rotational Speed $(\mathrm{rad} / \mathrm{s})$

$n=$ polytropic exponent

$\stackrel{g}{V}=$ volumetric flow

$$
\psi=\frac{g H}{\omega^{2} D^{2}}
$$

$N_{s}=\frac{\phi^{\frac{1}{2}}}{\psi^{\frac{3}{4}}}$

$\eta_{p}=\lambda \frac{\ln \left(P_{r}\right)}{\ln \left(T_{r}\right)}=\frac{\lambda}{\left(\frac{n-1}{n}\right)}$

$\eta_{s}=\frac{P_{r}^{\lambda}-1}{T_{r}-1}=\frac{P_{r}^{\lambda}-1}{P_{r}^{\frac{\lambda}{\eta_{p}}}-1}$

$\dot{m}=\rho \stackrel{g}{V}$

$\Delta P=$ pressure increase across compressor

$\rho=$ Density $\left(\mathrm{kg} / \mathrm{m}^{3}\right)$

$g=$ acceleration of gravity

$k=$ Specific heat ratio $=5 / 3$ for $\mathrm{He}$

$\lambda=\frac{k-1}{k}=0.4$ 